\title{
Testing deceased organ donors for Trypanosoma cruzi : the UK experience
}

Inês Ushiro-Lumb, Mhairi Webster, Alan Kitchen

Microbiology Services, NHS Blood and Transplant, London NW9 5BG, UK

\section{Background}

- With more than 10 million affected individuals, Chagas disease is the most common cause of heart failure and a frequent cause of intestinal problems in Latin America

- Trypanosoma cruzi infection is curable if treatment is initiated soon after infection

- In the UK, SaBTO recommends that donors with risk for $T$. cruzi infection be tested for specific antibodies (Ab)

- Since 2009 , less than $0.1 \%$ of the approximately 2.2million blood donations/year required T. cruzi Ab screening, with a positive rate of $0.016 \%$ (2 donors in 2009).

- Current annual positivity rate amongst blood donors is $0 \%$

- Centralised screening of deceased organ donors for $T$. cruzi $A b$ was introduced by NHSBT in October 2014 and is performed in the National Transfusion Microbiology Reference Laboratory (NTMRL)

\section{Methods}

- Requests submitted for T. cruzi Ab screening from deceased organ donors in England, $N$ Ireland and Wales between July 2014-Oct 2016 were reviewed for data collection

- Initially the Biokit Chagas Antibody® microplate assay and subsequently the Abbott Architect Chagas $\AA$ assay were used for screening, both as per the manufacturer's instructions

\section{Results}

- Risk of exposure to T. cruzi was identified in $77 / 4500$ potential organ donors (1.69\%)

- Reasons for testing are summarised in table 1

- The commonest indication for testing was travel to potentially endemic areas $(67 \%)$, whilst residency in endemic areas accounts for $32 \%$ of requests

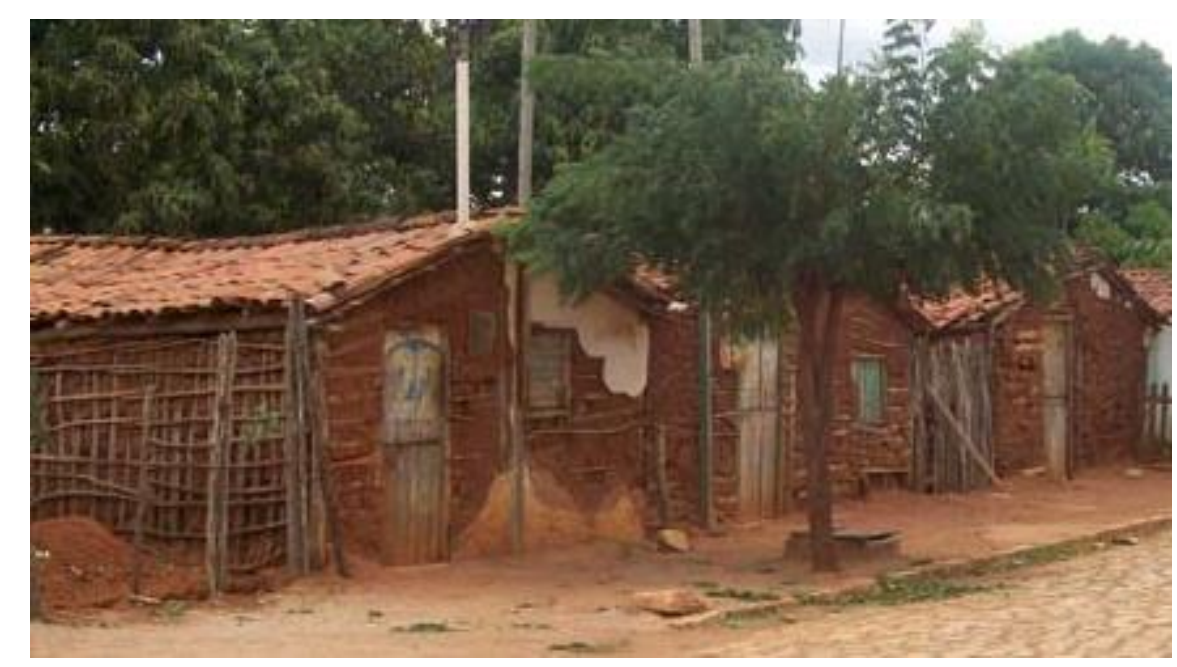

Fig 1: Migration routes from Latin America and estimation of the total number of infected individuals in non-endemic countries (with permission, Nature vol 465, 2010)

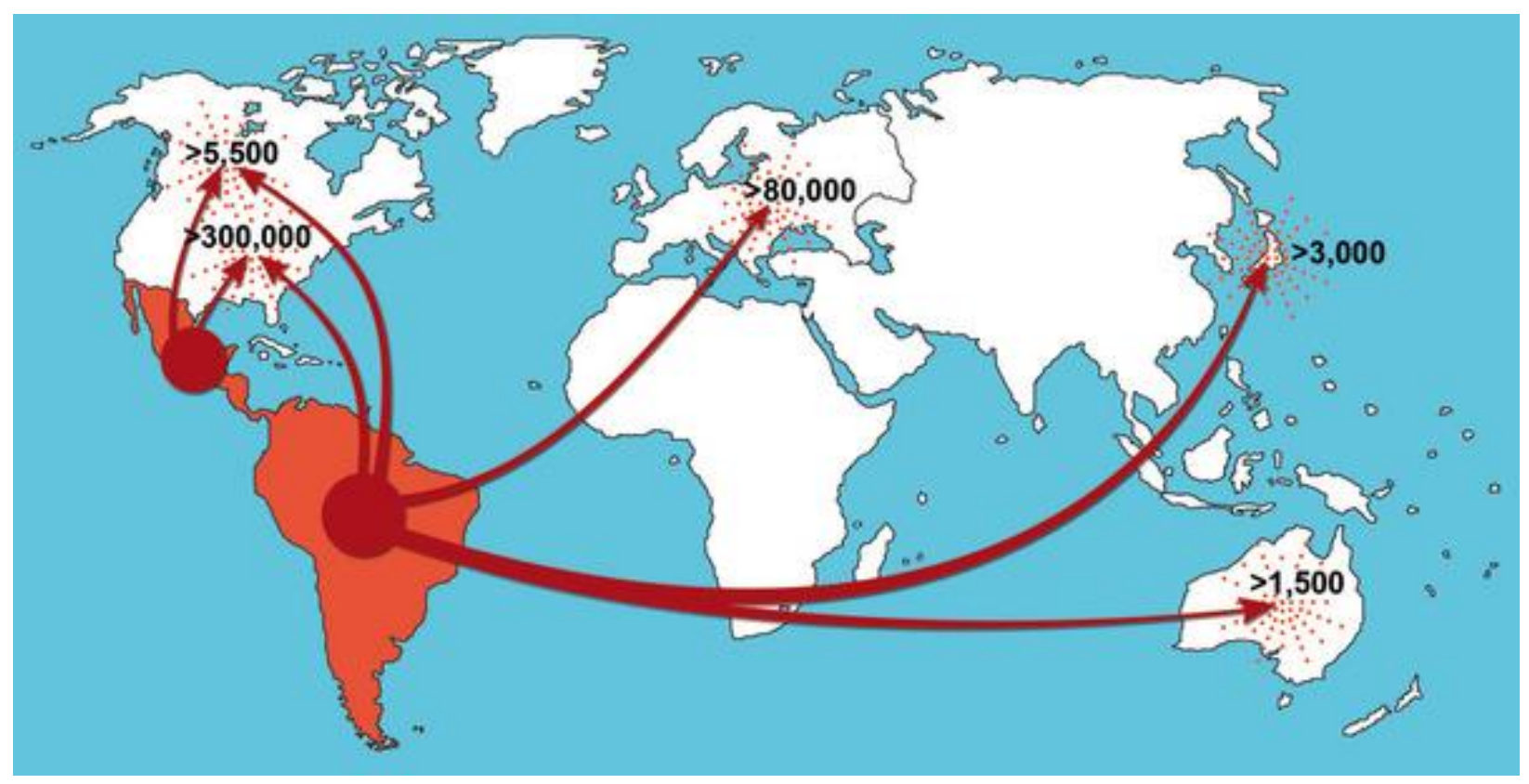

Table 1: Identified risk and indication for $T$ cruzi antibody screening

\begin{tabular}{|c|c|c|}
\hline Risk for T cruzi infection & $\mathbf{n}$ & $\%$ \\
\hline Born in endemic area & 15 & 19 \\
\hline Mother born* in endemic area $^{*}$ & 3 & 3.9 \\
\hline UK born visited endemic area & 49 & 64 \\
\hline UK born lived in endemic area & 5 & 6.5 \\
\hline $\begin{array}{c}\text { non UK born** visited endemic } \\
\text { area }\end{array}$ & 3 & 3.9 \\
\hline $\begin{array}{c}\text { non UK born } \\
\text { area lived in endemic }\end{array}$ & 2 & 2.6 \\
\hline Donors tested (total) & 77 & \\
\hline
\end{tabular}

*donor not born in endemic area

${ }^{* *}$ non endemic country

\section{Discussion and Conclusion}

- Initial identification of risk is based largely on donor demographics and broad travel information collected from the next of kin

- True risk of infection is associated with well defined factors and prolonged risk of vector exposure in rural, endemic or hyper endemic areas

- Donor or family history of conditions compatible with Chagas disease may be present and must be noted

- The risk of $T$. cruzi in our organ donor population remains low, reflecting the composition of migrant populations in the UK and our deceased donor pool

- In this setting, emphasis has been on detailed donor history, with post-donation screening; where high risk is identified, microbiological advice must be sought before donation

- Consideration for pre-donation testing requires suitable, appropriately validated tests, with specialist advice and laboratory familiarity with assay performance, so that results can correctly inform donor status 